\title{
Editorial
}

\section{Improving QoS and QoE for Mobile Communications}

\author{
Pedro Merino, ${ }^{1}$ Maria G. Martini, ${ }^{2}$ Raimund Schatz, ${ }^{3}$ \\ Lea Skorin-Kapov, ${ }^{4}$ and Martin Varela ${ }^{5}$ \\ ${ }^{1}$ Technical High School of Telecommunications Engineering, University of Málaga, Campus de Teatinos, 29071 Málaga, Spain \\ ${ }^{2}$ Faculty of Science, Engineering, and Computing, Kingston University London, Surrey KT1 2EE, UK \\ ${ }^{3}$ Telecommunications Research Center Vienna (FTW), 1220 Vienna, Austria \\ ${ }^{4}$ Faculty of Electrical Engineering and Computing, University of Zagreb, Unska 3, 10000 Zagreb, Croatia \\ ${ }^{5}$ VTT Technical Research Centre of Finland, P.O. Box 1100, 90571 Oulu, Finland
}

Correspondence should be addressed to Pedro Merino; pedro@lcc.uma.es

Received 1 August 2013; Accepted 1 August 2013

Copyright (c) 2013 Pedro Merino et al. This is an open access article distributed under the Creative Commons Attribution License, which permits unrestricted use, distribution, and reproduction in any medium, provided the original work is properly cited.

The proliferation of fast, cheap, and ubiquitous network access, in particular on mobile devices, has significantly changed the way users access online services on a day-to-day basis. Along with the increased availability of an always-on Internet, new technologies such as LTE have enabled the use of all types of services, including those that, such as video streaming, come with stringent requirements in terms of network performance and capacity demands. Consequently, the issue of adequately supporting all those users and all those services is a nontrivial one for network operators, as users expect certain levels of quality to be upheld.

In this special issue, we explore the relation between the Quality of Service (QoS) that operators monitor and manage and the Quality of Experience (QoE) that the users actually get. A good understanding of this relation is critical for network and service providers alike in order to properly provision and truly enhance their offerings so that end users receive the quality levels that satisfy or even delight them while at the same time enabling the efficient use of network resources.

The paper "Dealing with energy QoE trade offs in mobile video," authored by F. Liberal et al., addresses the transmission of scalable video over mobile networks. In particular, the focus is on the trade-off energy versus quality: depending on the specific type of service and network scenario, end users and/or operators may decide to choose among different energy versus quality combinations. In order to deal with this trade-off, the paper first proposes a single-objective optimization that is able to minimize the energy consumption with the constraint of a minimum acceptable quality. In order to reflect the fact that the same increment of energy consumption may result in different increments of visual quality, a multiobjective optimization is also proposed, with the associated utility function. Finally, in order to reduce complexity, a heuristic algorithm is proposed.

"Angry apps: the impact of network timer selection on power consumption, signalling load, and web QoE," authored by C. Schwartz et al. discusses the trade-off between energy consumption in smartphones and the generated signaling traffic in the mobile network. They use measurements made in public $3 \mathrm{G}$ networks to characterize the impact of inactivity timers in the network in energy consumption for different kinds of applications: bandwidth insensitive, interactive, and background applications. With this information, the authors study how the network operator could optimize the timers to keep the quality of service of a given application. However, such optimizations could also provoke more signaling traffic due to frequent connection reestablishments, and the authors discuss some of the mechanisms that try to resolve such conflicts. Finally, they specifically study the impact of the timer settings on QoE for web browsing.

In their paper "Survey and challenges of QoE management issues in wireless networks," S. Baraković and L. Skorin-Kapov provide a state-of-the-art survey on research in the field of QoE management for wireless networks. By identifying and discussing the key aspects and challenges of QoE modeling, monitoring, adaptation, and optimization from a wide interdisciplinary perspective, the paper enables a 
better understanding of QoE (and its relationship to QoS) as well as the process of its management in converged wireless environments. In particular, their work focuses on questions of what QoE influence factors to control, where to implement control mechanisms (e.g., network, client device), when to invoke them, and finally how to provide such control mechanisms, with the ultimate goal of realizing QoE optimization strategies that balance end-user as well as network provider interests.

The paper "Towards a QoE-driven resource control in LTE and LTE-A networks" by G. Gómez et al. focuses on meeting QoE requirements from a mobile network operator point of view. The authors propose an architecture for achieving QoEdriven resource control in LTE and LTE-advanced networks, based on the introduction of a centralized QoE server responsible for collecting relevant performance indicators, making QoE estimations, and triggering potential actions towards standardized 3GPP Evolved Packet System entities. The authors identify key performance indicators at both the application and network levels to be used for QoE estimation across different types of applications and highlight potential use cases for their proposed approach.

Finally, the paper "Obtaining more realistic cross-layer QoS measurements: A VoIP over LTE Use Case," by F. J. Rivas et al., presents a real-time testbed enabling realistic cross-layer measurements over LTE. This testbed enables the correlation of information at different layers, from service and IP levels to radio and protocol parameters. The analysis of the interlayer dependencies will support the identification of optimal settings for the radio access network and service parameters. New cross-layer optimization strategies could be suggested based on the observations provided. As a use case, VoIP over LTE is addressed.

\section{Acknowledgments}

This special issue cannot be produced without the effort of all the authors that responded to the call for papers and the work of the external reviewers. We are grateful to all of them.

Pedro Merino

Maria G. Martini

Raimund Schatz

Lea Skorin-Kapov

Martin Varela 

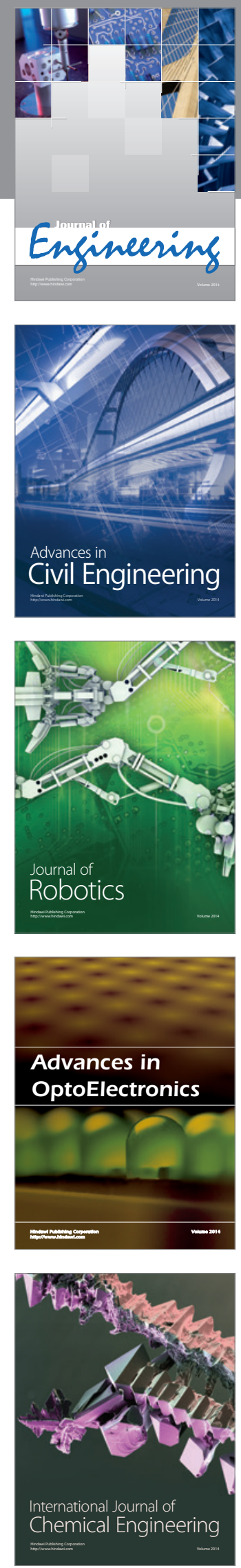

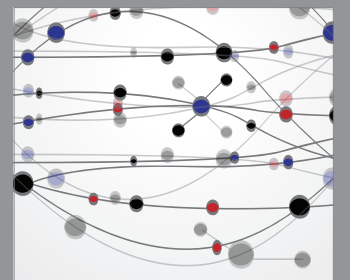

The Scientific World Journal
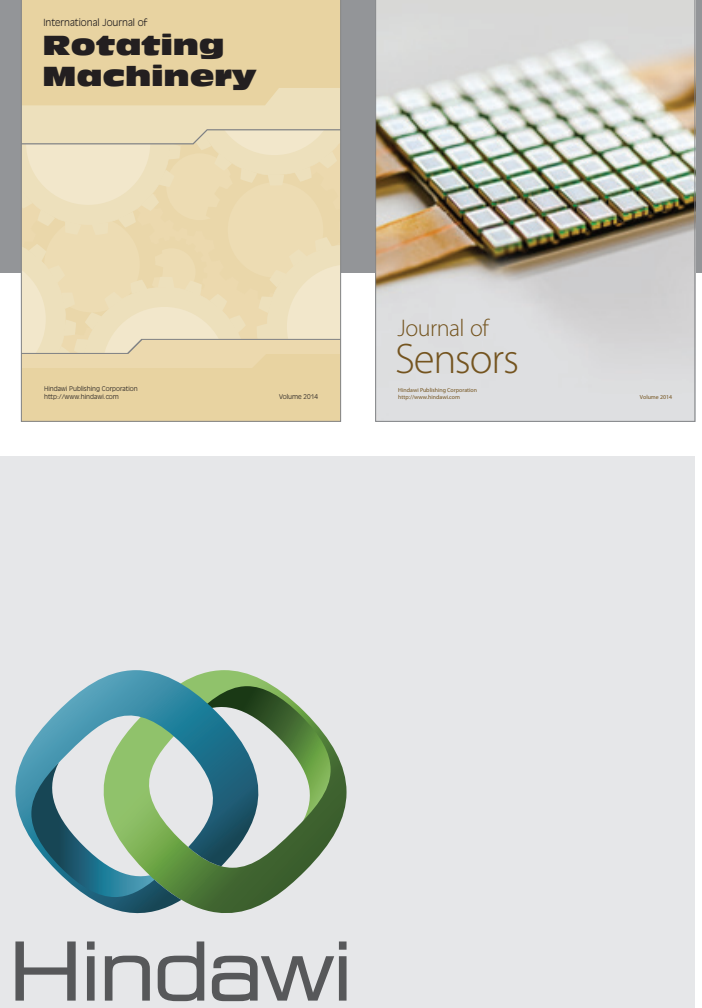

Submit your manuscripts at http://www.hindawi.com
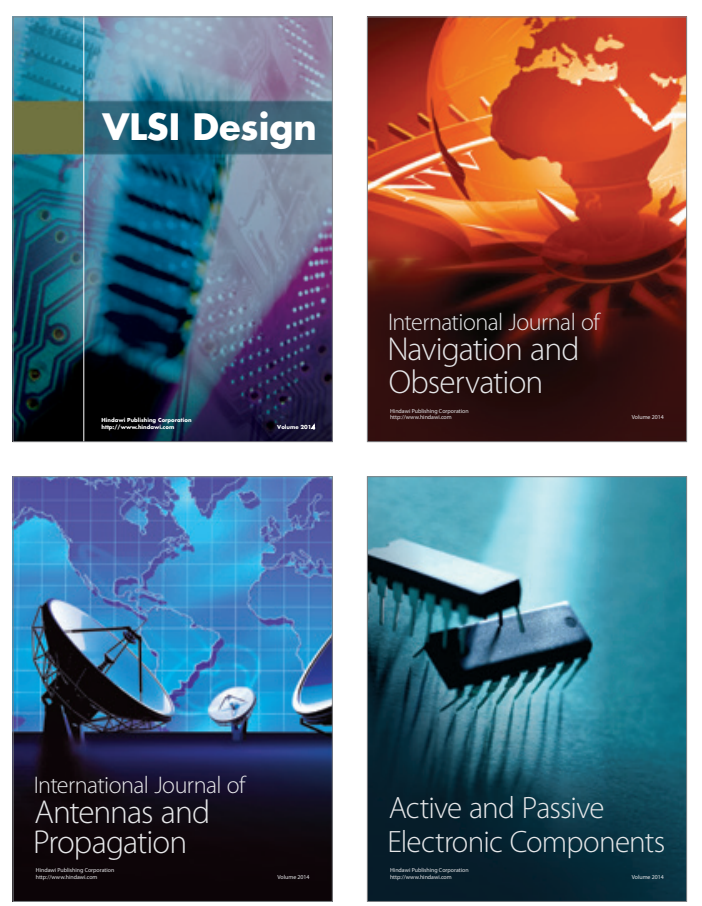
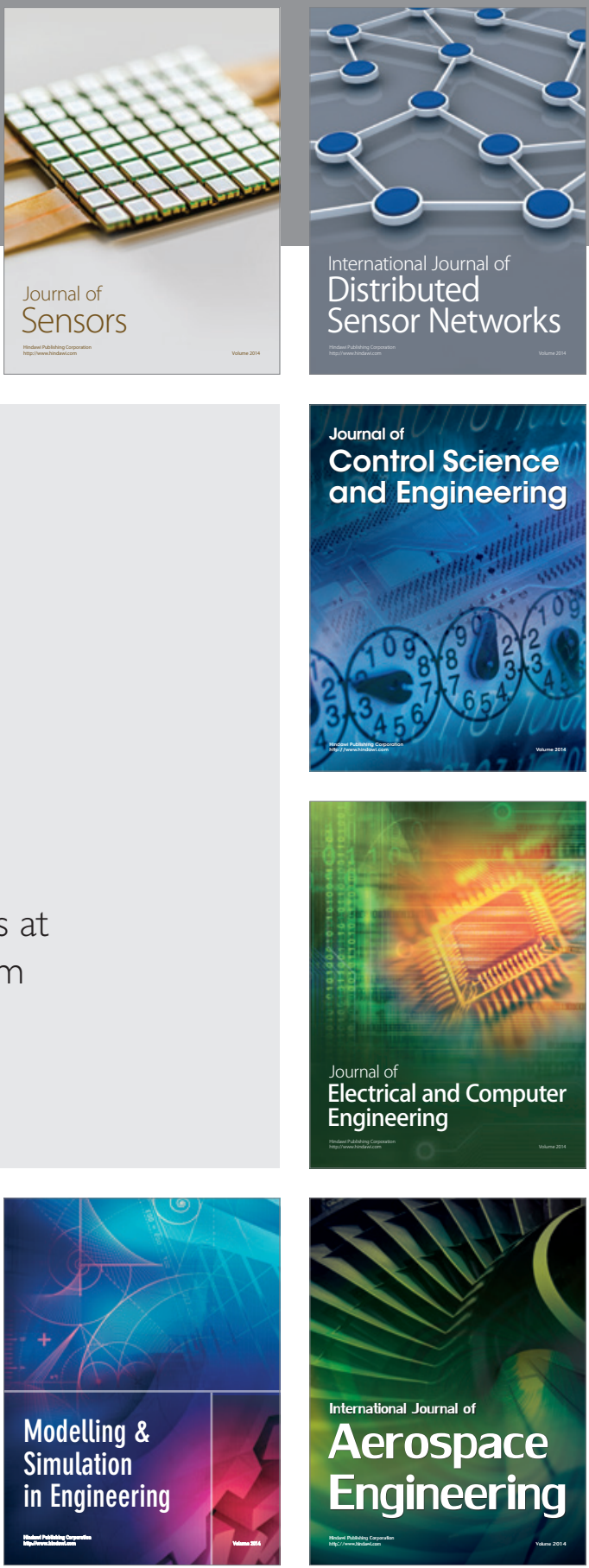

Journal of

Control Science

and Engineering
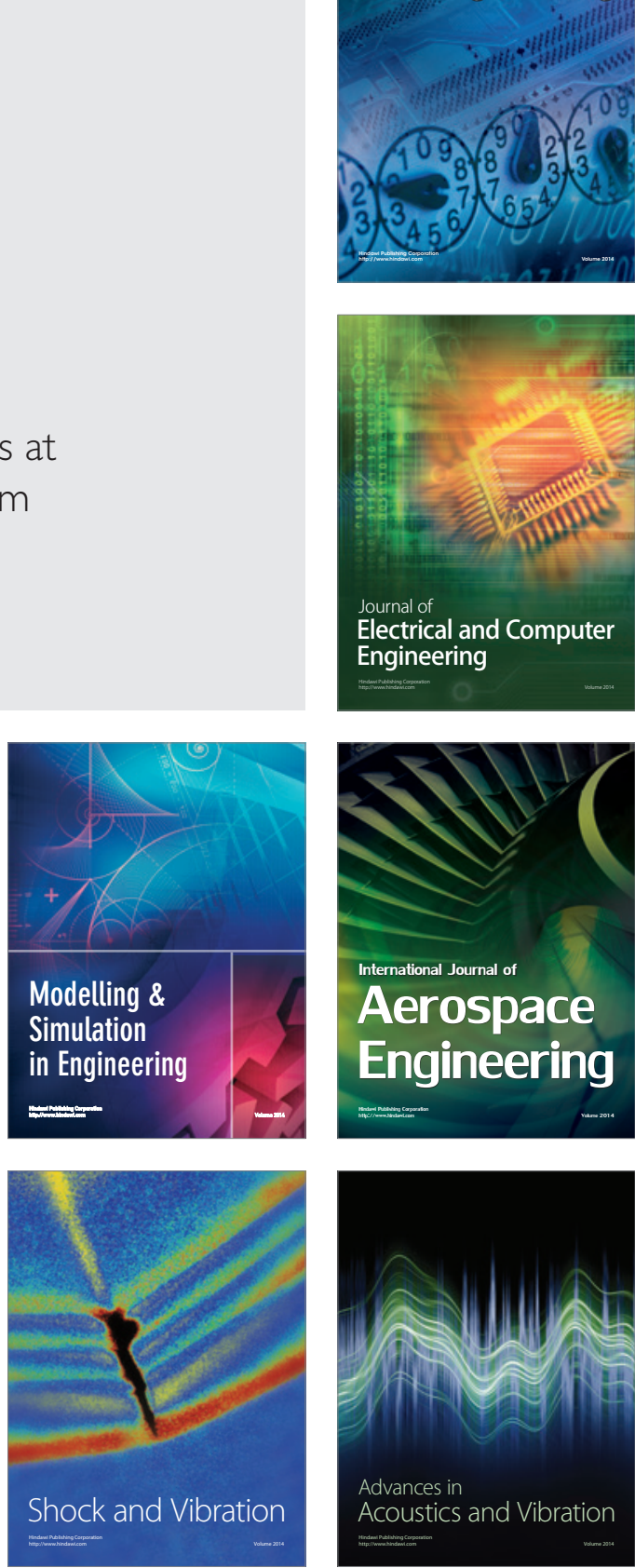